\title{
The Characteristics Analysis of Gear Box Fault Vibration Signal Xin-Tao JIAO
}

\author{
South China Normal University, 510631, Guangdong, China
}

Keywords: Gear box, Distributed fault, Centralized fault, Vibration signal.

\begin{abstract}
Gearbox is a critical component of mechanical equipment. Centralized fault and distributed fault are the two typical faults of gearbox. Both faults have their own features. In order to know them better, the characteristics in the time and frequency domain of the vibration signal were analyzed in detail. It was concluded that it was difficult to judge the type of faults only by the spectrum. Some simulation signals were used to verify the conclusions.
\end{abstract}

\section{Introduction}

Gear box is an important part of mechanical equipment and it is often used for transferring movement and power [1-3]. In modern large machinery, once there is gear fault, it may cause serious consequences [4-5].

The common faults of gear box can be divided into two categories [6]: distributed fault and centralized fault. The causes of these two kind faults are different, and their consequences are also different. In order to understand the fault characteristics of the gear box better and take good prepare for the gear box fault diagnosis, it is necessary to study the features of the gear box fault signal.

\section{The Vibration Feature of Gearbox}

During the meshing, the number of the teeth involved in the meshing changes from one pair to two pairs, then it changes back to one pair again. This meshing transformation alternating single and double pairs of teeth places a cyclical impact on the gear and causes the gear meshing vibration, which can be expressed as[6,7]:

$$
x(t)=\sum_{m=0}^{M} x_{m} \cos \left(2 \pi m f_{z} t+\varphi_{m}\right)
$$

Where ${ }^{x(t)}$ is the vibration signal, $f_{z}$ means the gear meshing frequency, ${ }^{x}$ and $\varphi_{m}$ denote the amplitude and phase of the $m$ th order gear meshing frequency component respectively.

\section{The Fault Characteristic of Gearbox}

The typical gear faults can be divided into two categories: distributed fault and centralized fault.

\section{Distributed Fault}

The distributed fault is often caused by the manufacturing or installation error. It may also be caused by wearing in using. Tooth profile error, even wear, the misalignment of the shaft and the unbalance of the shaft are the common typical distributed faults.

When there is a distributed error in the gear box, the amplitude of the vibration signal will change. The amplitude modulation function is shown in Equation (2). When the fault gear runs with the shaft one circle, the fault will appear once. So $a_{m}(t)$ is a periodic function with the rotating cycle of the shaft as the period.

$$
a_{m}(t)=\sum_{K=0}^{N}, A_{m} \cos \left(2 \pi k f_{n} t+a_{m, k}\right)
$$


In Equation (2), $A_{m, k}$ and $a_{m, k}$ are the amplitude and phase of the $\mathrm{k}^{\text {th }}$ order component in the $\mathrm{m}^{t h}$ amplitude modulation function respectively, $f_{n}$ is the rotational frequency of the shaft.

The stable modulate signal could be expressed as follows:

$$
\begin{aligned}
y_{s m}(t) & =\sum_{m=0}^{M} x_{m}\left[1+a_{m}(t)\right] \cos \left[2 \pi m f_{z} t+\varphi_{m}\right] \\
& =\sum_{m=0}^{M} x_{m}\left[1+\sum_{k=0}^{N} A_{m, k} \cos \left(2 \pi k f_{n} t+\alpha_{m, k}\right)\right] \cos \left(2 \pi f_{z} m t+\varphi_{m}\right)
\end{aligned}
$$

According to the trigonometric function transformations and the properties of the discrete Fourier transform (DFT), it can be found there are many couples of modulation sidebands in the spectrum of signal $y_{s m}(t)$. In which, the carrier frequencies are the meshing frequency and its octaves, the modulation frequencies are the rotational frequency and its octaves.

After being filtered by the band-pass filter with proper bandwidth, the rest component of $y_{s m}(t)$ is listed in Equation (4). In the filtering, the central frequency of the filter was set at the main frequency $m f_{z}$.

$$
y_{m}(t)=x_{m}\left[1+A_{m}, 1 \cos 2 \pi f_{n} t\right] \cos \left(2 \pi m f_{z} t\right)
$$

Letting $m=1, x_{m}=1, A_{m, 1}=1, f_{n}=20 \mathrm{~Hz}, f_{z}=2000 \mathrm{~Hz}$, there is:

$$
y_{s m}(t)=[1+\cos (2 \pi 20 t)] \cos (2 \pi 2000 t)
$$

The waveforms in time and frequency domains of the signal listed in Equation (5) were shown in Figure 1 . In the analysis, the sampling frequency is $8192 \mathrm{~Hz}$ and the sampling number is 1024 . The small window located in the right of Figure 1 (b) shows the partial enlarged view of the spectrum around $1500 \mathrm{~Hz}$. In this view, it can be found that the spectrum of the stable modulation signal is centered with the carrier frequency, $1500 \mathrm{~Hz}$, and the interval of the modulation sideband is $20 \mathrm{~Hz}$.

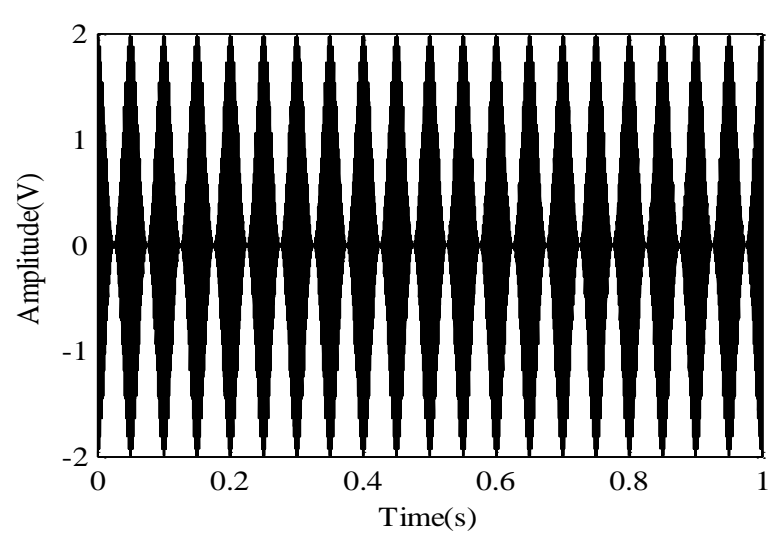

(a) Time waveform

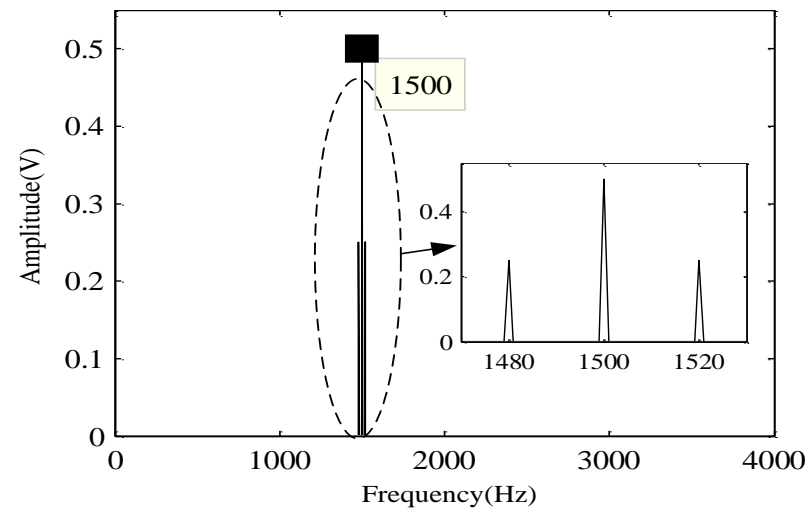

(b) Frequency waveform

Figure 1. Stationary modulation signal

According to the analysis, it can be concluded that the vibration signal of distributed fault has the following features:

(1) In the time domain, there is no impact vibration signal;

(2) In the frequency domain, there is modulation. The carrier frequencies are the meshing frequency and its octaves, the modulation frequencies are the rotational frequency of the shaft and its octaves. 


\section{Centralized Fault}

The centralized fault is often caused by some factors in using. The pitting, the peeling and the broken are all the typical common centralized faults. When the centralized fault appears in the gear box, there will be an impact modulation component in the vibration signal:

$$
y_{i m}(t)=\sum_{k=-\infty}^{\infty} x_{i m}\left(t-k T_{n}\right)
$$

Where

$$
x_{i m}(t)=A e^{-2 \pi \frac{\zeta}{\sqrt{1-\zeta^{2}}} f_{0} t} \cos \left(2 \pi f_{0} t\right)
$$

In Equations (6) and (7), ${ }^{T_{n}}$ is the period of the impact, $f_{0}$ is the carrier frequency, and $A$ is the amplitude.

Denoting the DFT of $x_{i m}(t)$ as $X_{i m}(\omega)$, according to the property of DFT, the DFT of $y_{i m}(t)$ can be described as follows:

$$
\begin{aligned}
Y_{i m}(\omega) & =\sum_{k=-\infty}^{\infty} X_{i m}(\omega) e^{-j \omega k T_{n}} \\
& =X_{i m}(\omega) \sum_{k=-\infty}^{\infty} e^{-j \omega k T_{n}} \\
& =X_{i m}(\omega) \sum_{k=-\infty}^{\infty} \delta\left(\omega-k \omega_{n}\right)
\end{aligned}
$$

Where $\omega_{n}$ is $2 \pi / T_{n}$,

$$
f(t) \delta\left(t-t_{0}\right)=f\left(t_{0}\right) \delta\left(t-t_{0}\right)
$$

According to the property of unit impulse function $\delta(t)$ shown in Equation (9), $Y_{i m}(\omega)$ equals to the superposition of $X_{i m}(\omega)$ at a series of discrete points, where $\omega$ equals $k \omega_{n}$. In $Y_{i m}(\omega)$, the central frequency is $f_{0}$, and the interval equals ${ }^{1 / T_{n}}$. The waveforms of $y_{i m}(t)$ in time and frequency domains were shown in Figure 2. In the simulation, the parameters $\zeta, f$ and $T_{n}$ are set to $0.02,2000 \mathrm{~Hz}$ and 0.05 s respectively. The small window located in the right of Figure 2 (b) shows the partial enlarged view of the spectrum around $2000 \mathrm{~Hz}$. From this view, it can be found easily that the spectrum is consisted of a series of discrete spectral lines. The central frequency is $2000 \mathrm{~Hz}$, and the interval equals $1 / T_{n}$, which is $20 \mathrm{~Hz}$.

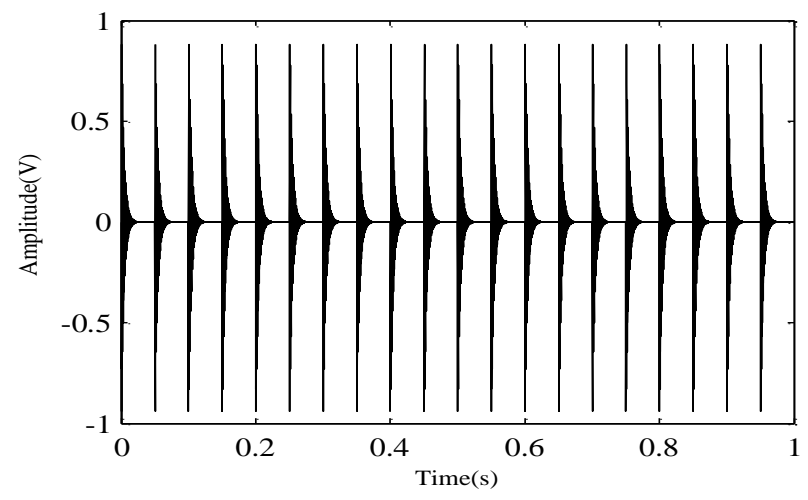

(a) Time domain waveform

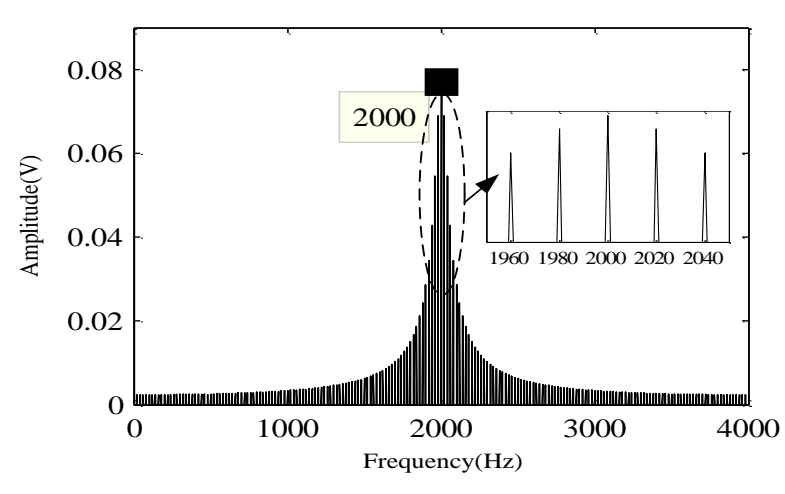

(b) Frequency domain waveform

Figure 2. Impact modulation signal 
As the positions of the centralized faults are relatively concentrated, the influence on the gear integral rigidity is relatively limited. The impact vibration only occurs when the faulty gear teeth are meshing, and the vibration signal shows as impact modulation, which can be described by Equation (6). When the other teeth are meshing, the faulty teeth will have no influence on the vibration signal, which can be expressed as meshing vibration shown in Equation (1). From this analysis it can be found when there is centralized fault in the gearbox, the vibration signal is consisted with two parts: the meshing vibration and the impact modulation.

A signal compounded with Equations (1) and (6) for simulating the vibration signal of the centralized fault was analyzed. The spectrum was shown in Figure 3. In the simulation, the meshing frequency $f_{z \text { is }} 1500 \mathrm{~Hz}$. As is known from the analysis expressed above, the spectrum of the impact modulation signal is always consisted of a series of discrete spectral lines, and the interval equals the modulation frequency. According to the relationship between the meshing frequency and the rotational frequency of the shaft, it can be inferred that the meshing frequency must equal the integer multiple of the rotational frequency. Therefore, in this series of discrete spectral lines, there must be one corresponding to the meshing frequency.

In Figure 3(a), when the centralized fault is more serious, the impact energy is so large that the spectrum of the impact modulation component extends to the meshing frequency. From the local enlarged drawing in Figure 3(a), it can be found, at the both sides of the meshing frequency, there are discrete spectral lines with equal interval. This characteristic is same with that of the stable modulation signal spectrum shown in Figure 1. For this, when analyzing this kind of spectrum, it's very easy to make a misunderstand that there is modulation in the signal with the meshing frequency and rotational frequency as the carrier frequency and modulation frequency respectively, and get a wrong conclusion there are distributed errors. But actually it isn't the case. In Figure 3(b), at the early stage of the centralized fault the impact energy is relatively small, the spectrum of the impact modulation component is concentrated around the natural frequency. At other points, it can not be found due to the decay. There are no discrete spectral lines with equal interval at the two sides of the meshing frequency, which is consistent with the frequency characteristic of the normal gear meshing. According to this kind of spectrum, it is easy to draw a wrong conclusion.

According to the analysis, the vibration signal of the centralized fault has some characteristics listed as follows:

In time domain, there are regular impact vibrations;

In frequency domain, the meshing frequency and its harmonics are obvious;

(3) When the centralized fault is serious, it may cause big impact and natural frequency modulation. In this kind of modulation, the carrier frequency may be the natural frequency of the gears, the shaft or the gear housing. The modulation frequency can be the rotational frequency and its octaves of the shaft where the fault gear installed, which could be found in the demodulation spectrum. The modulation sideband is often wide. On the contrary, at the early stage of centralized fault, the gear damage is relatively small. The impact of the meshing is so small that the impulse is often submerged by the other components of the vibration signal. That makes the characteristic in time domain weak, and only the meshing frequency and its octaves could be found in the spectrum. 


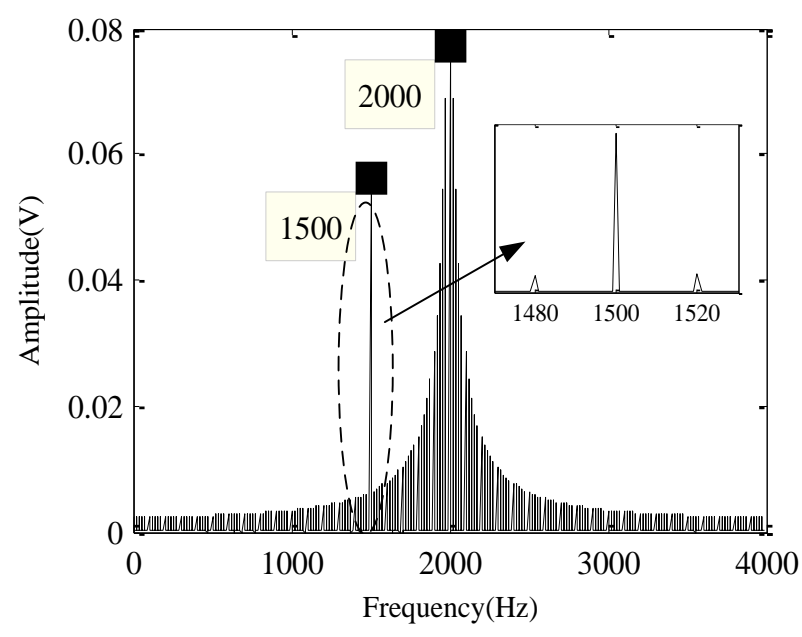

(a) Impact energy is large

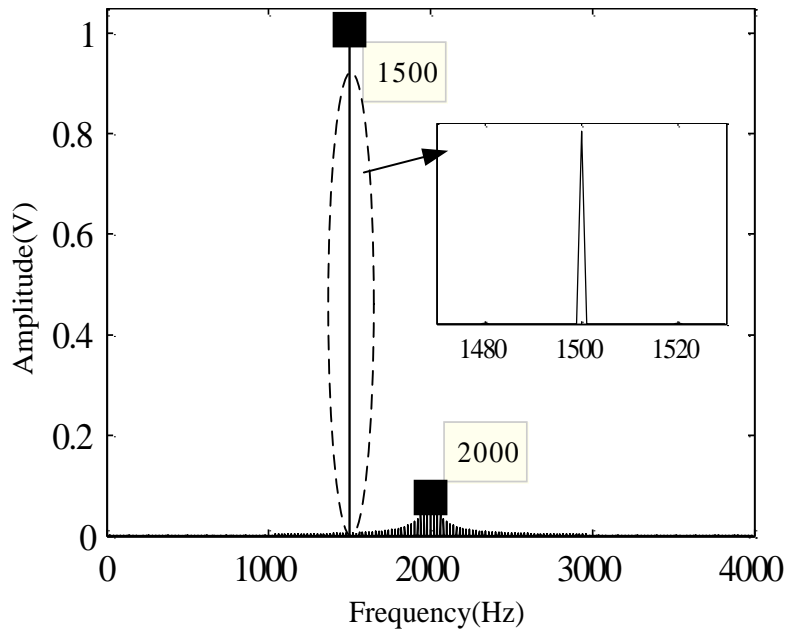

(b) Impact energy is small

Figure 3. Waveform of the centralized fault

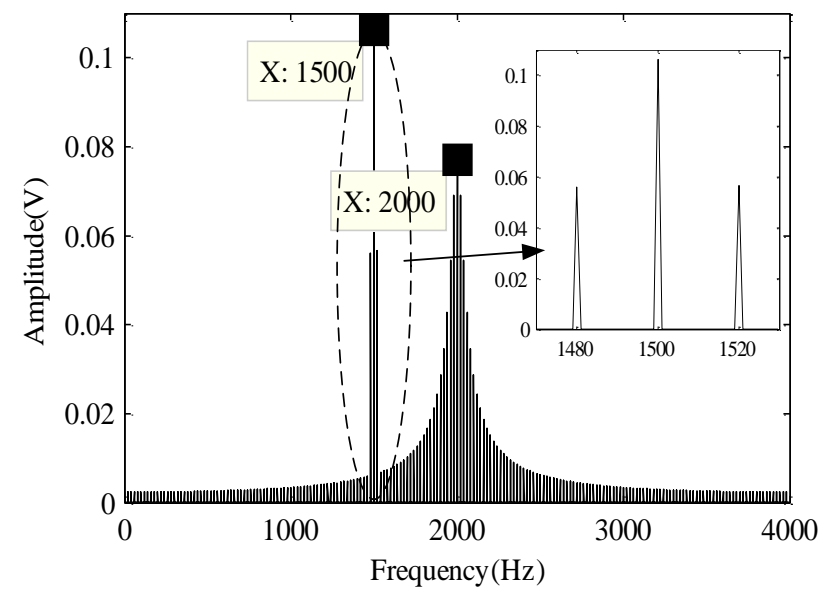

Figure4. Waveform of the vibration signal existing two faults

When there exits distributed fault and centralized fault in the gearbox simultaneously, the spectrum of the vibration signal is shown in Figure 4. The spectral characteristic is consistent with that shown in Figure 3(a), when there is only large centralized fault. Contrasting the spectral characteristic of these two signals, it can be concluded that it is impossible to judge the type of fault only by the spectrum.

\section{Conclusion}

In summary, the severity of centralized fault in the gear box has great influence on the characteristic of the vibration signal. When the fault is serious, the frequency characteristic near the meshing frequency is consistent with that of the distributed fault. At the early stage of centralized fault, the impact energy is small and the frequency characteristic is consistent with that of the normal gear. When there are these two types of faults in the gear box, the frequency characteristics are consisted with that there is only serious centralized fault. Therefore, it is difficult to determine accurately whether there is fault and what kind of fault exist in the gear box.

\section{Acknowledgement}

This work is support by the fund of SCNU (14KJ06) and the Natural Science Foundation of Guangdong (2016A030310436). 


\section{References}

1. F.Pacheco, J.V.Oliveira, R.V. Sánchez, M. Cerrada, Neurocomputing, 192,194(2016)

2. I. Antoniadou, G. Manson W.J. Staszewski, Mech. Sys. Sig. Proc.188, 64 (2015)

3 M. Bozca, Peter Fietkau, Mech and Mach Theory, 1599, 45(2010)

4. T. Praveenkumar M. Saimurugan, P. Krishnakumar, Proc. Eng,2092,97(2014)

5. B.Q. Chen, Z.S. Zhang, C. Sun, Mech. Sys. Sig. Proc, 275, 33 (2012)

6. K. Ding, W.H. Li, X.Y. Zhu. China Mach. Press (2005)

7. K. Ding, X.Y.Zhu, Y.H. Chen. J of Vib. and Shock, 7,20 (2001). 